\title{
Acute physiological responses on performance of Choy Lee Fut forms in amateur practitioners
}

\author{
Authors' Contribution: \\ A Study Design \\ B Data Collection \\ C Statistical Analysis \\ D Manuscript Preparation \\ E Funds Collection
}

\author{
Juan Manuel Cortell-Tormo ${ }^{A B C D}$, Miguel García-Jaén ${ }^{A B C D E}$, Roberto Cejuela ${ }^{B D}$, Iván \\ Chulvi-Medrano ${ }^{\mathrm{BD}}$, Carmen Manchado-López ${ }^{\mathrm{BD}}$, Julia Gómez-Ramos ${ }^{\mathrm{BD}}$
}

Area of Physical Education and Sports, Faculty of Education, University of Alicante, Alicante, Spain

Received: 16 November 2016; Accepted: 23 June 2017; Published online: 25 August 2017

AoBID: 11357

\section{Abstract}

Martial arts' forms are established sequences of offensive and defensive techniques representing simulated fights against fictitious opponents. Research has studied different acute physiological responses of form performance in other combat sports. However, in Choy Lee Fut (CLF) style, these responses remain unexplored. Therefore, the aim of this investigation was the knowledge about acute physiological responses in the performance of CLF form training session on the heart rate (HR), blood lactate concentration ( $\left.\mathrm{La}^{-}\right)$and serum creatine kinase concentration (CK) in amateur practitioners.

Material and Methods:

Ten male volunteers from a CLF amateur team participated in this study. HR, La-, and CK were measured in basal conditions, right after the empty-hand and bag form performance and 3-min after. CK post-exercise and 24 hours after $\mathrm{CK}_{24 \mathrm{~h}}$ were also registered. HR was measured through a cardio-tachometer, La- using a lactate analyser, and CK using a single-test clinical chemistry system.

Results: $\quad$ Study findings showed significant differences in HR responses between basal HR vs mean work HR, $(p=0.000)$ and maximal work HR, $(p=0.000)$. Likewise, significant differences were found in La- responses between basal $\mathrm{La}^{-}$vs post-exercise $\mathrm{La}^{-},(\mathrm{p}=0.000)$ and 3 -min after exercise $\mathrm{La}^{-},(\mathrm{p}=0.000)$. Finally, data also showed significant differences in $[C K] 24$-hour after exercise vs. post-exercise $(p=0.004)$ and basal values $(p=0.000)$; and between $\mathrm{CK}$ basal vs. post-exercise values $(\mathrm{p}=0.000)$.

Conclusions: $\quad$ Trial conditions elicited high HR and La- responses, which indicate that a session of the performance of CLF forms conforms to a high-intensity cardio-metabolic training session. Additionally, mild CK responses suggest small muscle damage. Therefore, this session could be recommended as an optimal exercise option for amateur practitioners seeking to improve their cardiorespiratory fitness.

Keywords: $\quad$ blood lactate $\bullet$ combat sports $\bullet$ creatine kinase $\bullet$ heart rate $\bullet$ kung-fu $\bullet$ male cardio-metabolic responses - martial arts

Copyright: $\quad$ C 2017 the Authors. Published by Archives of Budo

Conflict of interest:

Ethical approval:

Provenance \& peer review:

Source of support:

Authors have no financial affiliations (including research funding) or involvement with any commercial organisation that has a direct financial interest in the results of this study, as well as no conflicts of interest derived from the outcomes of this study.

The research was approved by the University Ethics Committee (UA-2016-09-17)

Not commissioned; externally peer reviewed

Miguel García-Jaén participated in this study supported by a pre-doctoral grant ACIF/2016/048 from the Generalitat Valenciana, Spain

Author's address:
Juan Manuel Cortell-Tormo, Area of Physical Education and Sports, Facultad de Educación; Carretera de San Vicente del Raspeig s/n 03690 San Vicente del Raspeig, Alicante, Spain; email: jm.cortell@ua.es 
Choy Lee Fut (CLF) - is a

Chinese martial art emerged as a branch from kung fu in the $19^{\text {th }}$ century. It has become popular combat sport as an effective selfdefence system, acquiring its status and techniques as a single style. CLF combines the martial arts techniques from various northern and southern Chinese kung fu systems, and it is considered an external style, combining soft and hard techniques, as well as incorporating a wide range of weapons as part of its curriculum.

Heart Rate (HR) - represents the speed of the heartbeat and it is measured by the number of poundings of the heart per unit of time, typically beats per minute (beats.min-1). The normal resting adult human heart rate ranges from 60-100 beats. $\mathrm{min}^{-1}$, and the actions. that can provoke changes include physical exercise, anxiety, stress, sleep, illness and various medicaments.

Blood Lactate (La-) - Appears in the blood as a result of anaerobic metabolism when oxygen delivery to the tissues is insufficient to support norma metabolic demands. Thus, Lalevel rises with the exercise of increasing intensity. Increased La- production coincides with cellular acidosis and remains a good indirect marker for cell metabolic conditions that induce metabolic acidosis.

Serum Creatine Kinase (CK) - The serum level of skeletal muscle enzymes is a marker of the functional status of muscle tissue and varies widely in both pathological and physiological conditions. An increase in these enzymes may represent an index of cellular necrosis and tissue damage following acute and chronic muscle injuries. The study of CK in sports medicine allows obtaining information on the state of the muscle.

Inbar et al. formula - $\mathrm{HR}_{\max }$ declined 0.685 beats.min ${ }^{-}$ 1.yers ${ }^{-1}(\mathrm{HR} \quad 205.8-0.685$ min $^{-1}$.yers $\left.{ }^{-1}\right)[36]$

Martial Arts - are systems of fight practices (practised for many reasons: selfdefence, competition, selfimprovement, physical health and fitness, mental and physical development) [37]

Randori - sparring in judo in which both participants practice attacking and defending [38].

\section{INTRODUCTION}

The Chinese martial arts are ancient forms of self-defence, and currently, many styles are practised all over the world not only for self-protection but also as a competitive sport and a form of exercise for health and fitness [1, 2]. In this sense, traditional martial arts styles such as karate, taekwondo, aikido, kung fu and jiu-jitsu, among other styles, have been becoming popular combat sports and have grown rapidly over the last years such as training methods worldwide [3, 4]. Within martial arts, kung fu was created in China around A.D. 520 [2]. Over the centuries, the movements of this art were refined, modified, and expanded, and throughout its course of development, many different branches or styles of KF were formed as different practitioners developed their techniques [2]. Within this expansion, Choy Lee Fut (CLF) style appeared and recently it has become a popular combat sport.

Although hundreds of styles exist at present, KF can essentially be classified into two disciplines: modern combat (sanshou) and formalised (taolu) kung fu [5]. Combat kung fu is characterised by regulated one-on-one sparring, whereas formalised kung fu is usually a set of choreographed movements, encompassing sub-categories of fist arts, weaponry, one-on-one practice and practice in groups [6]. Taolu is also commonly known as kung fu forms or systems, similar to katas in karate martial art. Kata, which means "form", has a conventional structure where athletes perform predetermined series of movements and techniques in known order against imaginary opponents, moving in several directions in space $[4,7]$. Form training aims to improve specific indicators, like technique, rhythm, power, the expressiveness of movements, etc. as close as possible to the ideal gesture [8]. This performance of forms requires motor control, rhythm, and movement differentiation capacities, and it demands a combination of dynamic and explosive movements [8]. Several studies have analysed the physical and physiological profile of karate athletes while performed these kata, and the information concerning performance analysis after a single session of karate has been well established $[9,10]$. Also, some researchers have studied physiological profiles in other sports combats, like kickboxing, wrestling, judo, and taekwondo [11, 12]. However, to date in kung fu martial art, there is a scarce research addressing this issue [11]. Moreover, to the best of our knowledge, there is a lack of research in CLF style, and the acute effects of form training session in this kung fu style remain uninvestigated.

Monitoring and determining the acute physiological responses of athletes in each sport is important to define aspects of physical performance that are essential not only to design a competitive training plan but also to avoid the undesirable overtraining syndrome $[11,13]$. These objective data of acute effects of exercise allow coaches to monitor the workload, to ensure that a specific and careful training elicits the desired effects on the athlete, both in wellness and health-related benefits as well as in improving their sportrelated performance $[13,14]$. Specificity is one of the physical training principles, which states that the specific exercise promotes adaptations in athletes' body and thus generating sport-specific effects of training. This principle is critical in training program design and establishes the necessity to distinguish specific physiological characteristics of one sport for specific training program design $[15,16]$. For that purpose, the determination of workload is necessary. In this sense, to determine the metabolic demands as well as the acute heart rate (HR) and blood lactate La- responses are key indicators, to evaluate the performance of forms in a single training session of combat sports $[14,17]$.

On the other hand, creatine kinase (CK) is a muscle enzyme whose serum concentration has been used as a biomarker of muscle damage after exercise and may indicate the status of the muscle cell membranes [18-21]. CLF is a kung fu style where forms are performed both in using empty-hand or in using a heavy bag or a wooden dummy with repetitive high-intensity actions and changes of directions. These movements impose an eccentric load on the limbs muscles that could result in extra muscular damage after a single training session. It is well documented that eccentric muscle contractions induce muscle damage, especially when performed in an unconditioned state [21, 22]. However, to the extent of our knowledge, CK blood concentration after the performance of form training session in CLF style have been uninvestigated, and currently, this question remains unclear.

Therefore, the aim of this investigation was the knowledge about acute physiological responses in the performance of CLF form training session on the heart rate (HR), blood lactate concentration ( $\mathrm{La}^{-}$) and serum creatine kinase concentration (CK) in amateur practitioners. 
It was hypothesised that the acute metabolic responses HR and La- will increase due to the training workload without concomitant increase of muscular damage CK.

\section{MATERIAL AND METHODS}

\section{Participants}

Ten volunteers of the CLF amateur regional team from the South of Spain took part in this study. All participants were male (mean \pm SD age $=30.6 \pm 8.13$ years; body mass $=75.7 \pm 7.11 \mathrm{~kg}$; height $=1.75$ $\pm 0.03 \mathrm{~m}$; training days per week $=2.7 \pm 1.33$ days). Experience levels of practitioners ranged from yellow fringe ( 2 participants with more than 2 years of practice); orange fringe (1 participant with more than 1 years of practice); blue fringe (4 participants from 4 to 7 years of practice); brown fringe (1 participant with 5 years of practice); to black fringe (2 participants with more than 7 years of practice). Participants were considered eligible for inclusion in this study if they were experienced with specific exercise technique and performed efficiently the different CLF forms selected for this study. Participants were excluded if their performance of CLF forms was not satisfactory, based on technique and intensity of exercises performed, they had any pathology or injury, or had an inability or unwillingness to complete the study procedures.

Each participant was informed about the study protocol, potential risks and benefits involved in the study, and signed a written consent form before participation. This investigation was confirmed to the standards set by the Declaration of Helsinki and was reviewed and approved by the University Ethics Committee (UA-2016-09-17).

\section{Experimental procedure}

All anthropometrical and physiological measurements were taken in laboratory conditions. Body mass was obtained to the nearest $0.1 \mathrm{~kg}$ using a balance beam (Avery Ltd. Model 3306 ABV), whereas height was measured using a stadiometer (Holtain Ltd.) to the nearest $0.5 \mathrm{~cm}$. Afterwards, participants were instrumented for telemetric HR assessment and informed about the La- and CK measurement processes. The HR was measured through a cardio tachometer (Suunto Ambit 3; Suunto. Oy, Vantaa, Finland), Lausing a lactate analyzer (Lactate Scout; SensLab. $\mathrm{GmbH}$, Leipzig, Germany) and finally, CK using a single-test clinical chemistry system (Reflotron Plus; Roche Diagnostics S.L. Sant Cugat del
Vallès, Barcelona). Capillary blood samples were collected from the earlobe in a seated position for the La- data collection. Likewise, for the CK data collection, blood samples were obtained from the index finger using capillary glass tubes and then were analysed using Reflotron system.

The tests were performed in the morning in a randomised order for participants on two separate days with a $48 \mathrm{~h}$ interval. Basal values of HR, La-, and $\mathrm{CK}$ were measured just before starting the warm-up and the study protocol. Post-exercise and 3-min post-exercise HR and La- were registered after the performance of each CLF form. CK was also registered post-exercise and $24 \mathrm{~h}$ after exercise trials. Exercise trials were carried out according to the pre-established study protocol.

Hence, before each test, the athletes were familiarised with the instrumentations and the procedures. Then, all participants were asked to perform a guided and standardised 10-min warm-up consisting of approximately 5 minutes of general warm-up with static and dynamic stretching and 5 minutes of sport-specific movements. After the warm-up, participants were started with the established exercise protocol. Participants randomly carried out several taolu of the CLF style, either using a heavy bag or performing empty-hand forms, at the hard or very hard intensity. During the performance of the different taolu, to obtain similar physiological stress levels between participants, the intensity of efforts was controlled by the values of rating of perceived exertion reported by the participants at the end of each taolu performed. Therefore, it was controlled that each participant performed the forms between values of "hard" and "very hard" exertion using the Borg's Scale (6-20) to validate each data measurement.

Then, during the experimental procedures, each participant performed the same Sah Bau Jong form on the heavy bag for four minutes long. On the other hand, based on their belt grade, and experience and knowledge in this martial art, each one performed one different empty-hand CLF form also for four minutes long. Therefore, participants executed in randomised order Ng Lun Ma, Sei Moon Kiu, Siu Mui Fa or Yee Jong Bot-Gwa Kuen empty-hand forms for four minutes and also Sah Bau Jong heavy bag form for other four minutes long, throughout overall trials. HR and La- were collected right after the end of each form and 3-min after the end of each performed form. After each HR and La- measurement, participants rested for 10 minutes. 
Finally, to complete the study protocol, each participant executed all the form sequence previously performed for 30minutes, resting ninety seconds between each form. Then, $\mathrm{CK}$ values were registered post-exercise and $24 \mathrm{~h}$ after each exercise trial. The total duration of the CLF form training session was 65 minutes approximately, within each trial of this investigation, including the data collection periods and excluding the 15 minutes of warm-up and 5 minutes of a cooldown. During the development of the trials, to ensure the appropriate effort of practitioners, different videos about kung fu sanshou and taolu were used motivating participants.

\section{Statistical analysis}

All statistical analyses were performed with SPSS 21.0 for Windows software (SPSS, Chicago, IL, USA). After checking the normality of the data (Kolmogorov-Smirnov), a general linear model with repeated-measures ANOVA was used to analyse the changes in the dependent variables studied (HR, La- and CK blood concentration). Bonferroni post hoc tests were used for pair-wise comparisons. Statistical significance was set at $\mathrm{p}<0.05$. All variables are reported as mean \pm SD.

\section{RESULTS}

\section{Heart Rate Outcomes}

The different obtained values of HR from study conditions were presented in Table 1. Significant differences were found between $\mathrm{HR}_{\text {bas }}$ vs $\mathrm{HRW}_{\text {mean }}$ ( $p=0.000)$ and $\mathrm{HR}_{\text {bas }}$ vs HRW $\max (p=0.000)$.

\section{Blood Lactate Concentration}

Also, the La- responses after trial conditions were $1.47 \pm 0.26 \mathrm{mmol} \cdot \mathrm{L}^{-1}$ from basal values $\mathrm{La}^{-}{ }_{\text {pre }}$;
$10.703 \pm 4.72 \mathrm{mmol} \cdot \mathrm{L}^{-1}$ from post-exercise values $\mathrm{La}^{-}{ }_{\text {post }}$ and $10.05 \pm 5.47 \mathrm{mmol} \cdot \mathrm{L}^{-1}$ from 3 -minute after exercise values $\mathrm{La}^{-}{ }_{3^{\prime}}$ (Figure 1). These results showed significant differences on $\mathrm{La}^{-}$ between basal values $\mathrm{La}^{-}{ }_{\text {pre }}$ and post-exercise values $\mathrm{La}^{-}{ }_{\text {post }}(p=0.000)$ and also significant differences between basal values $\mathrm{La}^{-}$pre and 3-minute after exercise values $\mathrm{La}^{-}{ }^{\prime}(\mathrm{p}=0.000)$. Finally, there were no significant differences on La- between $\left.\mathrm{La}^{-}{ }_{\text {post }}\right)$ and $\mathrm{La}^{-}{ }_{3^{\prime}}(\mathrm{p}=0.979)$.

\section{Blood Creatine Kinase Concentration}

Regarding CK, the obtained values were 88.26 $\pm 35.88 \mathrm{UL}^{-1}$ in $\mathrm{CK}$ basal values $\mathrm{CK}_{\text {basal }} ; 121.91$ $\pm 50.43 \mathrm{UL}^{-1}$ in post-exercise $\mathrm{CK}$ values $\mathrm{CK}_{\text {post }}$; and $192.60 \pm 67.60 \mathrm{UL}^{-1}$ in CK 24-hour values after exercise trials $\mathrm{CK}_{24 \mathrm{~h}}$ (Figure 2). These data showed significant differences from $\mathrm{CK}_{24 \mathrm{~h}} \mathrm{com}$ pared to $\mathrm{CK}_{\text {post }}(\mathrm{p}=0.004)$ and $\mathrm{CK}_{\text {basal }}(\mathrm{p}=0.000)$. Likewise, there were significant differences in blood $\mathrm{CK}$ concentration between $\mathrm{CK}_{\text {basal }}$ and $\mathrm{CK}_{\text {post }}(\mathrm{p}=0.000)$.

\section{DISCUSSION}

This study aimed to investigate the acute physiological responses on $\mathrm{HR}, \mathrm{La}^{-}$and $\mathrm{CK}$ after a training session on the performance of CLF empty-hand and bag forms in amateur practitioners. Results supported our initial hypotheses and showed that the reported magnitudes for $\mathrm{HR}$ and $\mathrm{La}^{-}$values were high, and the reported magnitude for $\mathrm{CK}$ values was mild. Hence, these data indicate that the cardio-metabolic workload of the trial was kept elevated whereas the muscle damage remained within normal values. Therefore, these findings highlight that the performance of CLF forms could confirm an optimal

Table 1. Heart rate (HR) obtained values from participants during study conditions.

\begin{tabular}{lllll}
\hline Variable & Min & Max & Mean & SD \\
\hline$H R_{\text {bas }}\left(\right.$ beats $\left.\cdot \mathrm{min}^{-1}\right)$ & 63.00 & 115.00 & 86.00 & 12.98 \\
\hline$H R_{\text {max }}\left(\right.$ beats $\left.\cdot \mathrm{min}^{-1}\right)$ & 177.03 & 191.42 & 184.68 & 5.20 \\
\hline$H R W_{\text {mean }}\left(\right.$ beats $\left.\cdot \mathrm{min}^{-1}\right)$ & 150.00 & 182.00 & 170.17 & 8.75 \\
\hline$H R W_{\text {max }}\left(\right.$ beats $\left.\cdot \mathrm{min}^{-1}\right)$ & 156.00 & 189.00 & 177.41 & 8.24 \\
\hline$H R_{\text {res }}\left(\right.$ beats $\left.\cdot \mathrm{min}^{-1}\right)$ & 63.40 & 127.73 & 98.68 & 16.64 \\
\hline$H R W_{\text {mean }}(\%)$ & 68.11 & 106.29 & 86.78 & 11.95 \\
\hline$H R W_{\text {max }}(\%)$ & 72.81 & 115.15 & 94.07 & 11.57 \\
\hline
\end{tabular}

$\mathbf{H R}_{\text {bas }}$ basal heart rate; $\mathbf{H R}_{\text {max }}$ formula of Inbar et al. [36]; $\mathbf{H R W}_{\text {mean }}$ mean of heart rate work; $\mathbf{H R W}_{\text {max }}$ maximal heart rate work; $\mathbf{H R}_{\text {res }}$ heart rate reserve $\left(H R_{\text {max }}-H R_{\text {bas }}\right)$. 


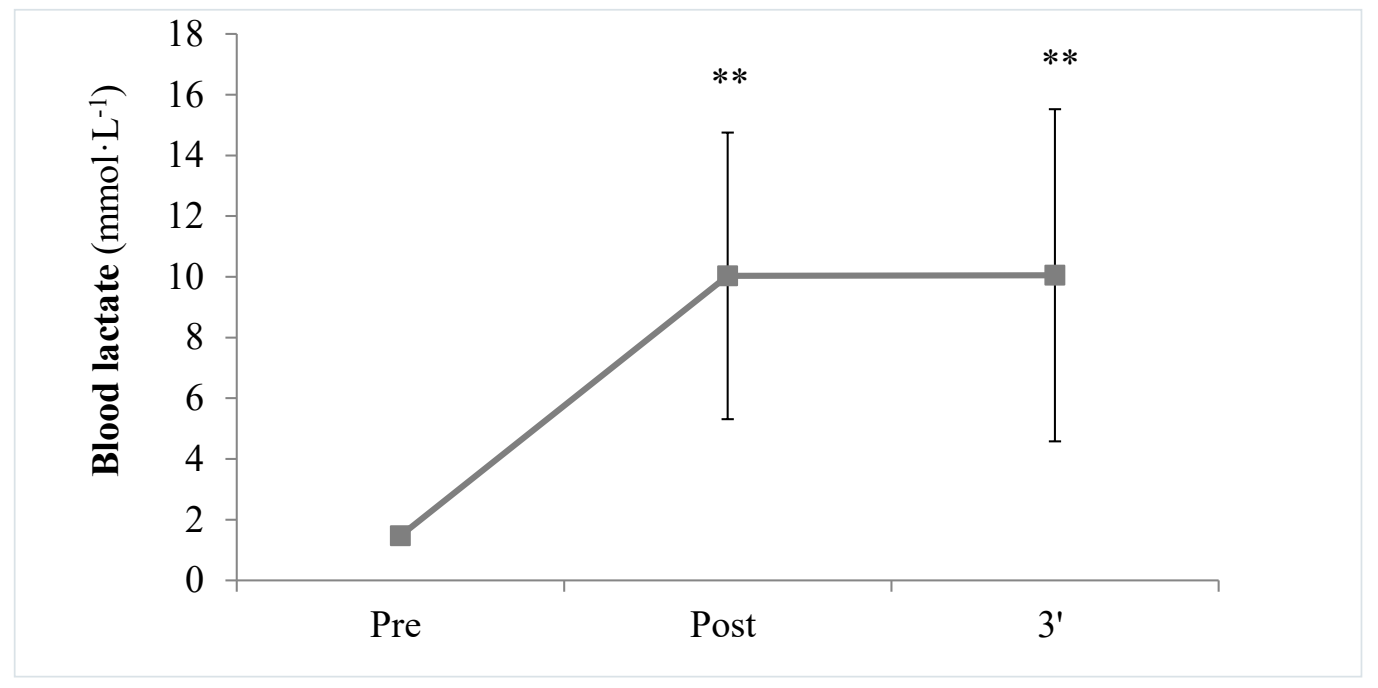

Figure 1. Comparison of $\mathrm{La}^{-}$basal values $\mathrm{La}^{-}{ }_{\text {pre, }}$, post-exercise values $\mathrm{La}^{-}{ }_{\text {post }}$ and 3-minute after exercise values $\mathrm{La}^{-}{ }_{3}$.

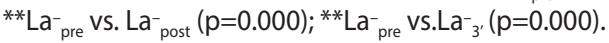

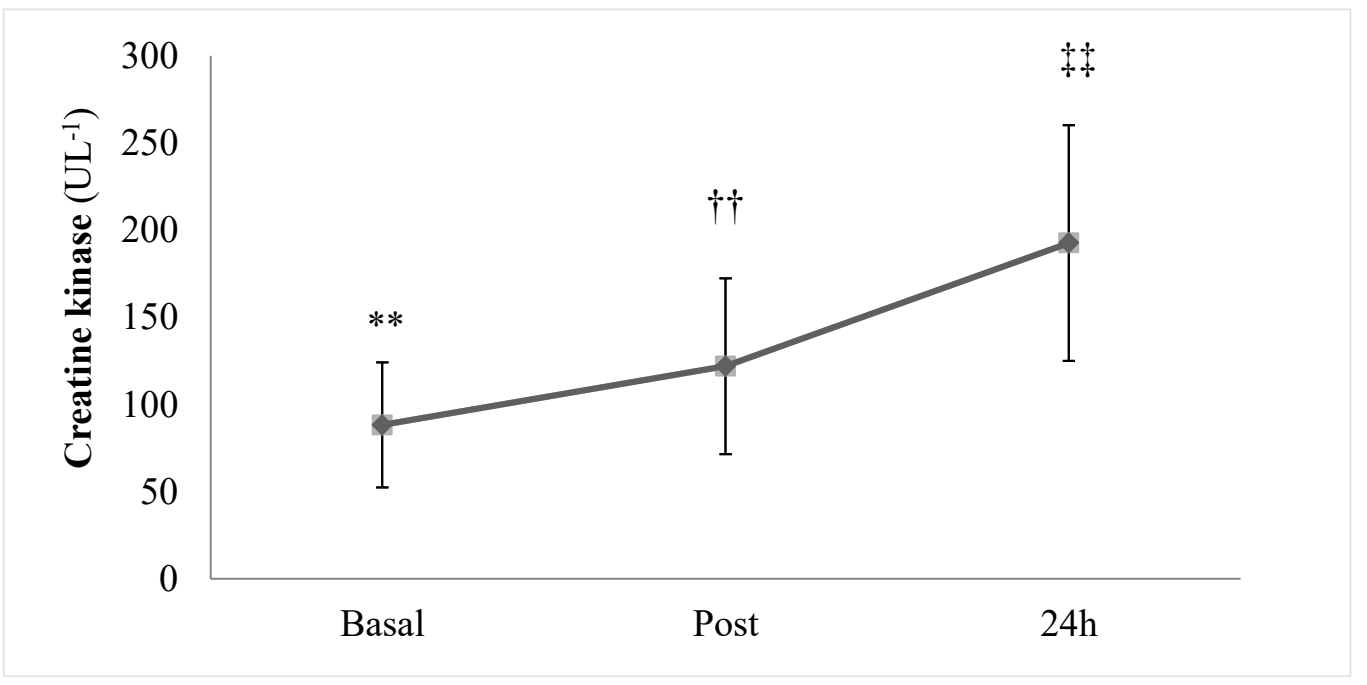

Figure 2. Comparison of $\mathrm{CK}$ basal values $\mathrm{CK}_{\text {basal }}$ post-exercise values $\mathrm{CK}_{\text {post }}$ and 24-hour values after exercise trials $\mathrm{CK}_{24 \mathrm{~h}}$. **Basal vs. post: $p=0.000 ; \dagger+$ post vs. $24 \mathrm{~h}: \mathrm{p}=0.004$; $\neq$ fbasal vs. $24 \mathrm{~h}: \mathrm{p}=0.000$.

cardio-metabolic training session, which could be recommended for enhancing the cardiorespiratory fitness of amateur practitioners.

To our knowledge, this is the first study designed to examine acute physiological responses on $\mathrm{HR}$, La- and CK in amateur CLF practitioners after a training session consisting of the performance of different empty-hand and bag CLF forms. Consequently, comparison of our results with other findings in related literature is limited because of this lack of CLF combat sports research and because of the scarce number of empirical studies analysing acute physiological responses in other similar combat sports that exist at present.
The present study showed that HR and La- values reached in trials were elevated. This cardiovascular and metabolic high response observed in participants indicates that the work intensity achieved on the performed session was vigorous [23], and the increase of HR and La- post-exercise values regarding baseline values was significant $(p<0.000)$. These findings are consistent with previous studies evaluating $\operatorname{HR}[14,24$, 25] and La- responses [14, 26-28] during combats or training sessions of other combat sports.

Regarding HR results, Ribeiro et al. [14] demonstrated in a study evaluating this physiological response in male athletes after the performance 
of form training session of modern Wushu that these athletes attained $89.1 \%$ of age-predicted maximum heart rate of work $\left(\mathrm{HRW}_{\max }\right)$. Other researchers [24, 25], who directly analysed the cardiovascular responses of male practitioners while performing specific techniques and forms from different kung fu styles, observed that the intensity of HR ranged between $64.4 \%$ and $70.5 \%$ of $\mathrm{HRW}_{\max }$. In our study, the values reached $84.63 \%$ of $\mathrm{HRW}_{\text {mean }}$, and $92.54 \%$ of $\mathrm{HRW}_{\max }$. These values are similar, or even slightly higher, than those showed in such three studies are. In this sense, the American College of Sport Medicine (ACSM) states that, according to the overload principle of training, it is necessary a minimum threshold of intensity to attain improvements in maximal oxygen uptake $\left(\mathrm{VO}_{2 \max }\right)$ and other physiological indicators [23]. This threshold of exercise intensity has a high variability of individual response, but ACSM has developed a classification based on different methods for estimation of relative exercise intensity for cardiorespiratory fitness. Within this classification, it has been established an interval of $77-95 \%$ of $\mathrm{HRW}_{\text {max }}$ as a range of vigorous intensity of exercise [23]. Considering this ACSM's Position Stand as well as previous studies analysed, it can be concluded that the intensity of the session performed in our study was vigorous, and would be suitable to develop and enhance cardiorespiratory fitness in amateur CLF practitioners.

La- concentration after the performance of CLF forms also reached high values. This increase was significant compared with baseline La- values $(p<0.000)$. Based on these data, we can conclude that there was a significant glycolytic pathway activation during the performance of CLF training session of forms in amateur practitioners, considering that anaerobic threshold has been already established in previous research in 4 $\mathrm{mmol} \cdot \mathrm{L}^{-1}$ [29]. Moreover, taking into account that this anaerobic threshold and the blood lactate curve are considered valid indicators of endurance performance [30], we can assume that the high La- and HR values achieved during trials indicate a high cardio-metabolic workload and, therefore, this performance of CLF forms could be a valid high-intensity training session to improve the endurance capacity in amateur practitioners. The obtained values in this study are similar with those described in other highly anaerobic-dependent combat sports like jiu-jitsu or judo, although these procedures did not conform to a form training session. Franchini et al. [26] reported $9.5 \pm 2.4$ $\mathrm{mmol} \cdot \mathrm{L}^{-1}$ during a male jiu-jitsu combat. Del Vecchio et al. (27) showed $10.2 \pm 1.5 \mathrm{mmol} \cdot \mathrm{L}^{-1}$ after a Brazilian jiu-jitsu male fight. Slightly higher $\mathrm{La}^{-}$values (11.2 $\left.\pm 3.0 \mathrm{mmol} \cdot \mathrm{L}^{-1}\right)$ were found in another study [28] with amateur male judo athletes. However, CLF form training session seems to be more anaerobic demanding activity compared with the performance of forms in modern Wushu, investigated by Ribeiro et al. [14]. The Lavalues showed in this study were $4.38 \pm 1.63$ and $5.15 \pm 1.07 \mathrm{mmol} \cdot \mathrm{L}^{-1}$ for Changquan and Daoshu forms, respectively, whereas La- values showed in our study were more elevated.

Regarding the obtained values of $\mathrm{CK}$, it can be concluded that the study conditions induced a mild increase in this marker of muscle damage on participants. Although our findings showed significant differences of $\mathrm{CK}_{24 \mathrm{~h}}$ compared to $\mathrm{CK}_{\text {post }}$ and $\mathrm{CK}_{\text {basal }}$, and also significant differences of $\mathrm{CK}_{\text {post }}$ compared to $\mathrm{CK}_{\text {basal, }}$, the results suggest that the magnitude of muscle fiber damage induced by performance of CLF training session of forms was mild, based on the small increase in CK blood concentration, which did not exceed the breakpoint of physiological values of CK serum release after exercise [31]. Therefore, based on previous research, we can assume that this CK serum elevation, occurred within 24 hours after trials, was physiological [31]. However, the results also suggest that full recovery from the training session could take more than one day, based on these significant differences in CK values $24 \mathrm{~h}$ after exercise trials regarding $\mathrm{CK}_{\text {post }}(\mathrm{p}=0.004)$ and $\mathrm{CK}_{\text {basal }}$ $(p=0.000)$. Nevertheless, it must be taken into consideration that because $\mathrm{CK}$ response depends on age, muscle mass, physical activity, and training experience, among other factors [31], and because the amateur participants of this study could be considered as a heterogeneous sample, conclusions regarding $\mathrm{CK}$ response could be relative, because these responses could present high variability with another more homogeneous experimental sample. Therefore, it would be necessary more research in this respect with CLF amateur practitioners to define more accurately these $\mathrm{CK}$ responses.

Since no previous studies have focused on muscle damage in CLF, comparison of the present study results can only be made to studies on other combat sports. The mean CK activity post-exercise in our study was $121.91 \pm 50.43 \mathrm{U}^{\mathrm{L}}-1$, which showed an increase of $38.13 \%$ regarding baseline CK activity. This increased rate is lower 
than that shown by Cordeiro et al. [32], who reported a significant increase of $78.23 \%$ regarding CK baseline activity after a session of training for combat in the Olympic KF $(218 \pm 198.09$ U.L-1 post-session CK versus $157 \pm 104.12$ U.L-1 pre-session CK). However, it must also be considered that the training loads and sports level of athletes in this study were not similar, and it could explain this higher $\mathrm{CK}$ response. Also, the physiological shift of CK activity post-exercise in our study is according with that observed by Umeda et al. [33], who demonstrated a physiological increase in CK (293.9 \pm 112.3 U.L-1) after standard judo training consisting of a warm-up, 70-min of randori and a cool-down. The structure and the total duration of our training session (65 minutes approximately, excluding the 15 minutes of warm-up and 5 minutes of a cool-down) was very similar to this study, and the physiological increase of CK level in both studies could be considered similar, although that the evident differences between both martial arts would limit these comparisons.

In contrast, Laskowski et al. [34] reported greater peak CK activity $\left(795 \pm 223{\left.\mathrm{U} . \mathrm{L}^{-1}\right)}^{-1}\right.$ and consequently higher muscle damage, after the last session of 3-day training cycle commonly used in judo practice. However, as the authors discussed in their study, the much higher values observed in this study could probably result from the accumulation of muscle damage induced by three consecutive training sessions, among other reasons. Therefore, analysing our results, we can conclude that one training session of CLF empty-hand and bag forms produce a physiological increase in the levels of muscular damage, and these values are mild. Thus, it could conform to a safe training session for amateur CLF practitioners with CK values within normal and physiological ranges [31].

In summary, our study showed a training session of forms shaped, constituted by a vigorous cardio-metabolic workload with low muscle damage, which could be a suitable and safe training option for CLF amateur practitioners who are seeking to reach the perfection of style and the improvement of their physical performance. Moreover, these findings could provide help to elaborate in the future the physiological profile of CLF style, although it would be necessary more studies with a more great and homogeneous sample. It is important to highlight that knowledge of the physiological and fitness profile of practitioners in a given sport is important to determine the capacities associated with the success in their performance [35].

Although the literature presents a variety of martial arts athlete profiles, amateur CLF practitioners' data have been lacking at the moment. Therefore, future research is necessary to complete the knowledge of the amateur practitioner of CLF style, on indicators, like anthropometric and nutritional profiles, muscle power and maximal dynamic strength, aerobic and anaerobic profile, flexibility, energetics, and finally reaction time, as it has already been investigated in other combat sports [10,11]. Also, new studies should be conducted to expand the knowledge of the physiological demands during a CLF form training performed in a couple, and during a CLF match, especially about multiple fights and competitive environments. Finally, it would be interesting to study the comparison of acute physiological responses of empty-hand versus bag CLF training session of forms, to find the higher physiological workload and set up the most suitable performance of forms for CLF practitioners. On the other hand, this study, despite to provide novel data regarding physiological stress in CLF, it also presented several limitations. Firstly, the relatively small sample size and the heterogeneity of the sample investigated (age, experience levels) could lead to underestimation of the results. Finally, comparison of our results with findings in literature was limited, because the empirical studies analysed were limited in number and quality, which demonstrates the lack of CLF combat sports research and highlights the need to develop and expand a more robust research in this widespread kung fu style.

\section{CONCLUSIONS}

The findings from the study of acute physiological responses in a training session on the performance of CLF forms showed high HR and $\mathrm{La}^{-}$values, which indicate that it conforms to a high-intensity training in amateur practitioners. Also, the mild CK values showed in the study suggest relatively small muscle damage values. Therefore, a training session on the performance of CLF forms could represent a vigorous and safe cardio-metabolic training session, and it could be recommended as an optimal exercise for enhancing cardiorespiratory fitness in amateur practitioners seeking to improve their physical fitness and their performance in this kung fu style. 


\section{ACKNOWLEDGMENTS}

The authors would like to thank José Luis Sala, president of KF LONG MEN PAI Club of Alicante (Spain) as well as the participants for their time and effort.

\section{REFERENCES}

1. Donovan OO, Cheung J, Catley M et al. An investigation of leg and trunk strength and reaction times of hard-style martial arts practitioners. J Sports Sci Med 2006; 5(CSSI): 5-12

2. Tsang TW, Kohn M, Chow CM et al. Health benefits of Kung Fu: a systematic review. J Sports Sci 2008; 26(12): 1249-1267

3. Zetaruk MN, Violán MA, Zurakowski D et al. Injuries in martial arts: a comparison of five styles. Br J Sports Med 2005; 39(1): 29-33

4. Vujkov S, Calleja-González J, Krneta Z et al. Physiological responses the organism of karate athletes specialists of kata and kumite during simulated competition. Arch Budo 2015; 11: 365-370

5. Sousa P, Marquezi M, Uliani R et al. Incidence of injuries to the lower limbs joints in kung fu athletes. Arch Budo 2010; 6(3): 137-142

6. Lu C. Modern wushu: When Chinese martial arts meet Western sports. Arch Budo 2008; 4: 37-39

7. Doria C, Veicsteinas A, Limonta E et al. Energetics of karate (kata and kumite techniques) in top-level athletes. Eur J Appl Physiol 2009; 107(5): 603-610

8. Invernizzi PL, Longo S, Scurati R. Analysis of heart rate and lactate concentrations during coordinative tasks: pilot study in karate kata world champions. Sport Sci Health 2008; 3(1-2): 41-46

9. Benedini S, Longo S, Caumo A et al. Metabolic and hormonal responses to a single session of kumite (free non-contact fight) and kata (highly ritualized fight) in karate athletes. Sport Sci Health 2012; 8(2-3): 81-85

10. Chaabène $H$, Hachana $Y$, Franchini $E$ et al. Physical and physiological profile of elite karate athletes. Sports Med 2012; 42(10): 829-843

11. Artioli GG, Gualano B, Franchini E et al. Physiological, performance, and nutritional profile of the Brazilian Olympic Wushu (kung-fu) team. J Strength Cond Res 2009; 23(1): 20-25

12. Sanchooli Z, Ghoochan FA, Ghoochan ZA et al. Determining Physiological Profile of National Iranian Wushu Team. Bull Env Pharmacol Life Sci 2014; 3: 69-72

13. Saw AE, Main LC, Gastin PB. Monitoring the athlete training response: subjective self-reported measures trump commonly used objective measures: a systematic review. Br J Sports Med 2016; 50(5): 281-291
14. Ribeiro JL, de Castro BO, Rosa CS et al. Heart rate and blood lactate responses to changquan and daoshu forms of modern wushu. J Sports Sci Med 2006; 5(CSSI): 1-4

15. Hewson DJ, Hopkins WG. Specificity of training and its relation to the performance of distance runners. Int J Sports Med 1996;17(3): 199-204

16. Hill DW, Leiferman JA, Lynch NA et al. Tempora specificity in adaptations to high-intensity exercise training. Med Sci Sports Exerc 1998; 30(3): 450-455

17. Berta LMÁ, Zagalaz JC, Brahim M et al. Las áreas funcionales implicadas en el entrenamiento especial competitivo del boxeo. Un estudio de casos. Retos 2014; (26): 71-74

18. Kleiner DM, Worley ME, Blessing DL. Creatine Kinase Response to Various Protocols of Resistance Exercise. J Strength Cond Res 1996; 10(1): 15-19

19. Rodrigues BM, Dantas E, de Salles BF et al. Creatine kinase and lactate dehydrogenase responses after upper-body resistance exercise with different rest intervals. J Strength Cond Res 2010; 24(6): 1657-1662

20. Gomes RV, Santos RC, Nosaka K et al. Muscle damage after a tennis match in young players. Biol Sport 2014; 31(1): 27-32

21. Moreira A, Nosaka K, Nunes JA et al. Changes in muscle damage markers in female basketball players. Biol Sport 2014; 31(1): 3-7

22. Clarkson PM, Hubal MJ. Exercise-induced muscle damage in humans. Am J Phys Med Rehabil 2002; 81(11): S52-S69

23. Garber CE, Blissmer B, Deschenes MR et al. American College of Sports Medicine position stand. Quantity and quality of exercise for developing and maintaining cardiorespiratory, musculoskeletal, and neuromotor fitness in apparently healthy adults: guidance for prescribing exercise. Med Sci Sports Exerc 2011; 43(7): 1334-1359

24. Schneider D, Leung R. Metabolic and cardiorespiratory responses to the performance of Wing Chun and T'ai Chi Chuan exercise. Int J Sports Med 1991; 12(3): 319-323

25. Jones MA, Unnithan VB. The cardiovascular responses of male subjects to kung fu techniques. Expert/novice paradigm. J Sports Med Phys Fitness 1998; 38(4): 323-329

26. Franchini E, Bezerra PL, Oliveira RSF et al.
Blood lactate concentration, heart rate and handgrip strength during a jiu-jitsu combat. Corpoconsciéncia 2005; 9(1): 35-44

27. Franchini E, Takito MY, Kiss MAPDM et al. Physical fitness and anthropometrical differences between elite and non-elite judo players. Biol Sport 2005; 22(4): 315-328

28. Del Vecchio FB, Bianchi S, Hirata SM et al. Morphofunctional analysis of Brazilian JiuJitsu practitioners and study of temporality and quantification of motor actions in the modality. Mov Percep 2007; 7(10): 263-281

29. Heck H, Mader A, Hess G et al. Justification of the 4-mmol/I lactate threshold. Int J Sports Med 1985; 6(3): 117-130

30. Faude O, Kindermann W, Meyer T. Lactate threshold concepts: how valid are they? Sports Med 2009; 39(6): 469-490

31. Brancaccio P, Maffulli N, Limongelli FM. Creatine kinase monitoring in sport medicine. Br Med Bull 2007; 81-82: 209-230

32. Cordeiro EM, Gomes ALM, Guimarães M et al. Hematological and biochemical alterations originating from the combat training in of Olympic Kung fu athletes. Fit Perform J 2007; 6: 255-261

33. Umeda T, Yamai K, Takahashi I et al. The effects of a two-hour judo training session on the neutrophil immune functions in university judoists. Luminescence 2008; 23(1): 49-53

34. Laskowski R, Ziemann E, Olek RA et al. The effect of three days of judo training sessions on the inflammatory response and oxidative stress markers. J Hum Kinet 2011; 30: 65-73

35. Skidmore BL, Jones MT, Blegen M et al. Acute effects of three different circuit weight training protocols on blood lactate, heart rate, and rating of perceived exertion in recreationally active women. J Sports Sci Med 2012; 11(4): 660-668

36. Inbar O, Oten A, Scheinowitz M et al. Norma cardiopulmonary responses during incremental exercise in 20-70-yr-old men. Med Sci Sport Exerc 1994; 26(5): 538-546

37. Biernat E, Boguszewski D. The level of physical activity of the working inhabitants of Warsaw practising martial arts and combat sports. Arch Budo 2015; 11: 69-77

38. Budō: The Martial Ways of Japan. Tokyo: Nippon Budokan Foundation; 2009

Cite this article as: Cortell-Tormo JM, García-Jaén M, Cejuela R et al. Acute physiological responses on performance of Choy Lee Fut forms in amateur practitioners. Arch Budo 2017; 13: 235-242 